\title{
A Rat Model of Progressive Nigral Neurodegeneration Induced by the Parkinson's Disease-Associated G2019S Mutation in LRRK2
}

\author{
Julien Dusonchet, ${ }^{1}$ Olexiy Kochubey, ${ }^{2}$ Klodjan Stafa, ${ }^{3}$ Samuel M. Young Jr, ${ }^{4}$ Romain Zufferey, ${ }^{1}$ Darren J. Moore, ${ }^{3}$ \\ Bernard L. Schneider, ${ }^{1}$ and Patrick Aebischer ${ }^{1}$ \\ ${ }^{1}$ Neurodegenerative Studies Laboratory, ${ }^{2}$ Laboratory of Synaptic Mechanisms, and ${ }^{3}$ Laboratory of Molecular Neurodegenerative Research, Brain Mind \\ Institute, École Polytechnique Fédérale de Lausanne, 1015 Lausanne, Switzerland, and ${ }^{4}$ Max Planck Florida Institute for Integrative Biology and \\ Neuroscience, Jupiter, Florida 33458
}

The G2019S mutation in the leucine-rich repeat kinase 2 (LRRK2) gene is the most common genetic cause of Parkinson's disease (PD), accounting for a significant proportion of both autosomal dominant familial and sporadic PD cases. Our aim in the present study is to generate a mammalian model of mutant G2019S LRRK2 pathogenesis, which reproduces the robust nigral neurodegeneration characteristic of PD. We developed adenoviral vectors to drive neuron-specific expression of full-length wild-type or mutant G2019S human LRRK2 in the nigrostriatal system of adult rats. Wild-type LRRK2 did not induce any significant neuronal loss. In contrast, under the same conditions and levels of expression, G2019S mutant LRRK2 causes a progressive degeneration of nigral dopaminergic neurons. Our data provide a novel rat model of PD, based on a prevalent genetic cause, that reproduces a cardinal feature of the disease within a rapid time frame suitable for testing of neuroprotective strategies.

\section{Introduction}

Mutations in the leucine-rich repeat kinase 2 (LRRK2) gene have emerged as the most common genetic determinant of Parkinson's disease (PD) (Berg et al., 2005; Healy et al., 2008). The most prevalent mutation in LRRK2, G2019S, accounts for 5-6\% of autosomal-dominant familial and $1-2 \%$ of sporadic PD cases (Di Fonzo et al., 2005; Gilks et al., 2005; Healy et al., 2008). The G2019S variant is found at frequencies as high as $40 \%$ of both familial and sporadic cases in specific populations such as Ashkenazi Jews (Ozelius et al., 2006) and North African Arabs (Lesage et al., 2006). Patients harboring the G2019S mutation are clinically indistinguishable from sporadic PD cases (Healy et al., 2008). The vast majority of LRRK2-linked cases display neuropathology typical of sporadic $\mathrm{PD}$, characterized mainly by a dramatic loss of dopaminergic neurons in the substantia nigra pars compacta (SNpc) (Giasson et al., 2006; Ross et al., 2006). A G2019S LRRK2 mammalian model, which recapitulates the hallmark nigral neurodegeneration, should therefore provide a powerful platform for evaluation of disease-modifying therapies

\footnotetext{
Received Sept. 29, 2010; revised 0ct. 25, 2010; accepted 0ct. 29, 2010.

This work was funded by the Michael J. Fox Foundation for Parkinson's Research (D.J.M. and P.A.), the Max Planck Society (S.M.Y.), Swiss National Science Foundation (D.J.M., Grant 310030_127478), and the École Polytechnique Fédérale de Lausanne (D.J.M. and P.A.). We thank Christel Sadeghi, Fabienne Pidoux, Vivianne Padrun, Cyrielle Deladoey, Liliane Glauser, Philippe Colin, and Nicolas Leuba for excellent technical assistance, Dr. Christopher Ross for the gift of the LRRK2 CDNAs, and Dr. RalfSchneggenburger for providing the platform for rAd production and for critical review of the manuscript.

Correspondence should be addressed to Patrick Aebischer, Neurodegenerative Studies Laboratory, Brain Mind Institute, Ecole Polytechnique Fédérale de Lausanne, EPFL SV BMI LEN, AI 2241, Station 19, 1015 Lausanne, Switzerland. E-mail: patrick.aebischer@epfl.ch.

D0I:10.1523/JNEUROSCI.5092-10.2011

Copyright $\odot 2011$ the authors $\quad 0270-6474 / 11 / 310907-06 \$ 15.00 / 0$
}

aimed at both familial and sporadic PD patients. Transgenic mouse models developed to date, however, fail to achieve significant levels of LRRK2 overexpression in the SNpc and consequently do not display dopaminergic neuronal loss ( $\mathrm{Li}$ et al., 2009, 2010; Melrose et al., 2010). Alternative gene delivery tools are therefore required to reproduce the progressive degeneration of nigral neurons associated with the G2019S mutation in PD.

\section{Materials and Methods}

Adenoviral vector construction and virus production

Second-generation E1, E3, E2a-deleted recombinant human serotype 5 adenoviruses ( $\mathrm{rAd}$ ) were generated (Zhou and Beaudet, 2000; Young and Neher, 2009). A modified version of the pDC511 shuttle plasmid (Microbix) was generated (Young and Neher, 2009) with an expression cassette consisting of the synthetic intron from pCI (Promega), the neuron-specific human synapsin-1 promoter (Kügler et al., 2001), a multiple cloning site, and the SV40 polyadenylation signal. Codonoptimized WT and G2019S human LRRK2 cDNAs with three tandem FLAG tags at the $\mathrm{N}$ terminus were provided by Dr. Christopher Ross (Johns Hopkins University, Baltimore, MD). Each cDNA was cloned in a two-step procedure into the multiple cloning site of the pDC511 shuttle plasmid, making use of a unique HindIII restriction site within the LRRK2 sequence. A pDC511-GFP plasmid was also generated. A modified version of the FLP, frt human Ad5 genomic plasmid (Microbix) and each pDC511 shuttle plasmid were cotransfected into E2acomplementing cells (E2T), and rAd virus production was performed according to standard protocol (Ng and Graham, 2002). Vector stocks were amplified from plaque isolates to guarantee homogeneity of the production. Final vector stocks were purified and concentrated using the Vivapure AdenoPACK 100RT kit (Sartorius). Viral titers of purified vector stocks were determined by $\mathrm{OD}_{260}$ measurements. The following titers were obtained, expressed as viral particles $(\mathrm{vp}) / \mu \mathrm{l}: 2.9 \times 10^{9} \mathrm{vp} / \mu \mathrm{l}$ for 
rAd-GFP, $2.3 \times 10^{9} \mathrm{vp} / \mu \mathrm{l}$ for rAd-LRRK2$\mathrm{WT}$, and $2.6 \times 10^{9} \mathrm{vp} / \mu \mathrm{l}$ for rAdLRRK2-G2019S.

\section{Stereotaxic unilateral injection into the} striatum of rats

Adult Wistar female rats (Charles River Laboratories) weighing $\sim 200 \mathrm{~g}$ were housed in $12 \mathrm{~h}$ light/dark cycle, with ad libitum access to food and water, in accordance with the Swiss legislation and the European Community Council directive (86/609/EEC) for the care and use of laboratory animals. For stereotaxic injections, the animals were deeply anesthetized with a mixture of xylazine/ketamine and placed in the stereotaxic frame (David Kopf Instruments). Recombinant Ad vectors were injected unilaterally in the striatum at six points, corresponding to two deposits along three needle tracts at the following anterior-posterior, mediolateral relative to bregma, dorsoventral relative to skull surface coordinates: $0.48 /-2.4 /-4.8$ and $-6.0 ; 0.48 /-3.2 /-4.8$ and $-6.0 ; 0.48 /-4.0 /$ -4.8 and -6.0 . Two microliters of virus at a concentration of $2.3 \times 10^{9} \mathrm{vp} / \mu$ l were injected per site using a $10 \mu \mathrm{l}$ Hamilton syringe with a 34 gauge blunt tip needle at a speed of $0.2 \mu \mathrm{l} /$ min, with an automatic pump (CMA Microdialysis). The more ventral site in each needle tract was injected first and the needle was left in place for 2 min before slowly moving to the more dorsal site in the same needle tract, after injection of which the needle was left for an additional $5 \mathrm{~min}$ before slowly being withdrawn.

\section{Western blot analysis}

Quantification of FLAG-LRRK2 expression levels. HEK 293T cells were infected with $3.2 \times$ $10^{9} \mathrm{vp}$ of rAd-LRRK2-WT or rAd-LRRK2G2019S ( $n=3$ per group) and harvested $48 \mathrm{~h}$ after infection in Triton extraction buffer (50 mm Tris- $\mathrm{HCl}, \mathrm{pH} 7.5,150 \mathrm{~mm} \mathrm{NaCl}, 2 \mathrm{~mm}$ EDTA, and 1\% Triton X-100) containing protease inhibitors (Roche Applied Science). rAdLRRK2-WT- and rAd-LRRK2-G2019S-injected rats were killed at $10 \mathrm{~d}$ after injection $(n=4$ per group). The SN and striata from injected and uninjected hemispheres were then rapidly dissected and homogenized in Triton extraction buffer containing protease inhibitors (Roche Applied Science). Western blot analysis on 50 $\mu \mathrm{g}$ of protein was performed with a mouse monoclonal antibody against FLAG (mouse IgG, 1:1000; Anti-FLAG M2, Sigma) and an anti-actin antibody to confirm equal protein loading (goat IgG, 1:1000; sc-1616, Santa Cruz Biotechnology). Detection and quantification were performed using the Odyssey Infrared Imaging System.

Soluble LRRK2 extraction and native PAGE/SDS-PAGE analysis. rAdLRRK2-WT and rAd-LRRK2-G2019S-injected rats were killed at $14 \mathrm{~d}$ after injection ( $n=4$ per group). The $\mathrm{SN}$ and striata from injected and uninjected hemispheres were then rapidly dissected and homogenized in $100 \mu \mathrm{l}$ of $50 \mathrm{~mm}$ HEPES, $\mathrm{pH}$ 7.4, containing protease and phosphatase inhibitors (Roche Applied Science). Lysates were centrifuged at $175,000 \times g$, and protein content in supernatants was determined by a BCA assay (Pierce). A fraction of the soluble extracts were combined with $4 \times$ reducing sample buffer (150 mm Tris- $\mathrm{HCl}, \mathrm{pH} 7.0,12 \%$ SDS, $6 \%$ $\beta$-mercaptoethanol, 30\% glycerol, and 0.05\% Coomassie Blue G-250) for SDS-PAGE analysis. Sixty micrograms of protein were loaded for

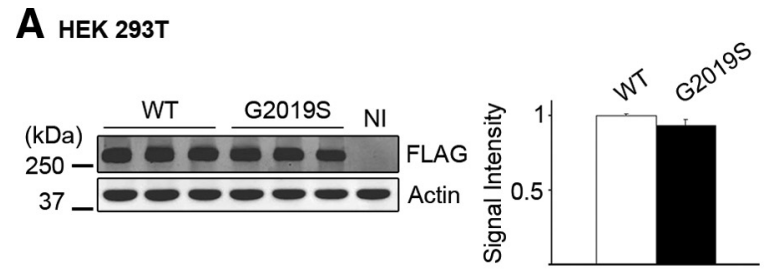

B wT
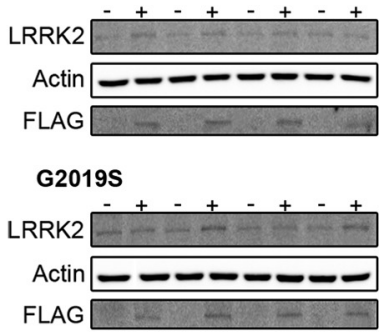

Fold LRRK2 overexpression (vs. uninjected side)

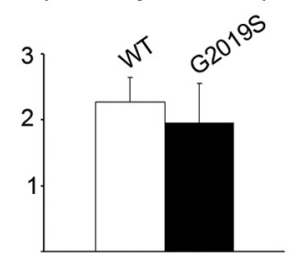

\section{C striatum}

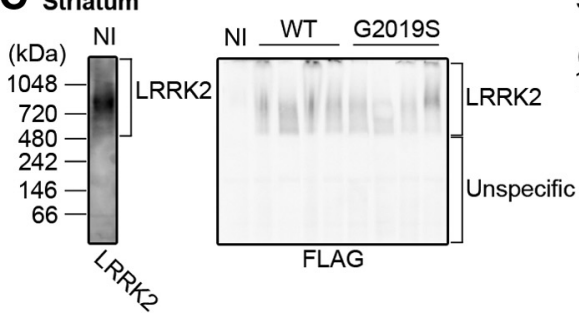

Substantia nigra
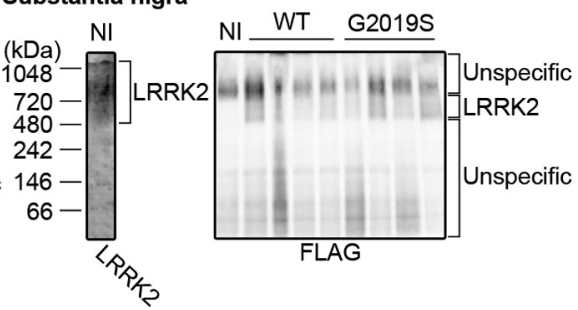

Figure 1. Comparable expression and oligomerization of WT and mutant G2019S LRRK2. A, Western blot analysis of FLAGLRRK2 expression. HEK 293T cells were infected with an equal amount of viral particles of rAd-WT-LRRK2 or rAd-G2019S-LRRK2 ( $n=3$ per group). A noninfected (NI) control was included. rAd-WT-LRRK2- and rAd-G2019S-LRRK2-injected animals were killed membranes were probed with anti-FLAG and anti-actin antibodies. Both vectors were found to drive equivalent levels of expression of FLAG-LRRK2 in vitro and in vivo, following densitometric analysis and normalization to actin levels. B, Fold human LRRK2 group). Protein extracts from uninjected ( - ) and injected (+) SN were separated by SDS gel electrophoresis. Membranes were bed with an anti-LRRK2 antibody that recognizes rodent and human LRRK2. The fold overexpression of human LRRK2 in each Further probing of the membrane with anti-FLAG antibody confirmed the rAd-driven expression of FLAG-LRRK2 in all injected SN. An average twofold overexpression of human LRRK2 was measured for both the WT and G2019S groups. C, Native PAGE analysis of endogenous and overexpressed human LRRK2. A fraction of the soluble protein extracts of dissected striata and SN were analyzed by native PAGE. Upon staining with an anti-LRRK2 antibody, endogenous LRRK2 in the striatum and SN was shown to assemble not detect any protein migrating at the size expected for monomeric LRRK2 neither in the $\mathrm{SN}$, nor in striatal extracts. Overexpressed G2019S LRRK2, detected by anti-FLAG immunostaining, showed a qualitatively similar higher-order distribution compared and endogenous LRRK2. Uninjected striatal and nigral extracts were loaded as controls for the specificity of the anti-FLAG signal. Despite the presence of a nonspecific band at $\sim 720 \mathrm{kDa}$ upon FLAG staining on SN lysates, we could clearly detec higher-order complexes between 480 and $720 \mathrm{kDa}$ for overexpressed LRRK2 in the SN.

each sample. The remaining fraction was used for loading on $4-16 \%$ bis-tris blue native polyacrylamide gels (Invitrogen) followed by overnight transfer to PVDF membranes. Equivalent sample volumes were loaded corresponding to $\leq 100 \mu \mathrm{g}$ of protein for striatal extracts and $\leq 90$ $\mu \mathrm{g}$ of protein for nigral extracts. Detection was performed using enhanced chemiluminescence (GE Healthcare). Antibodies used were antiFLAG-HRP (1:2000, mouse IgG; A 8592, Sigma) and anti-actin and rabbit monoclonal anti-LRRK2 (1:2000, c41-2/MJFF-2, kindly provided by the Michael J. Fox Foundation), which recognizes both rodent and human LRRK2. Densitometric analysis was performed using the NIH ImageJ software.

\section{Immunohistological analysis and quantification}

Tissue processing. For histological analysis, animals were killed at 10, 21, and $42 \mathrm{~d}$ after injection. $n=5$ /group/time point except $n=4$ for G2019S at $21 \mathrm{~d}$ (rat number 5 in this group was not included due to a technical 
A
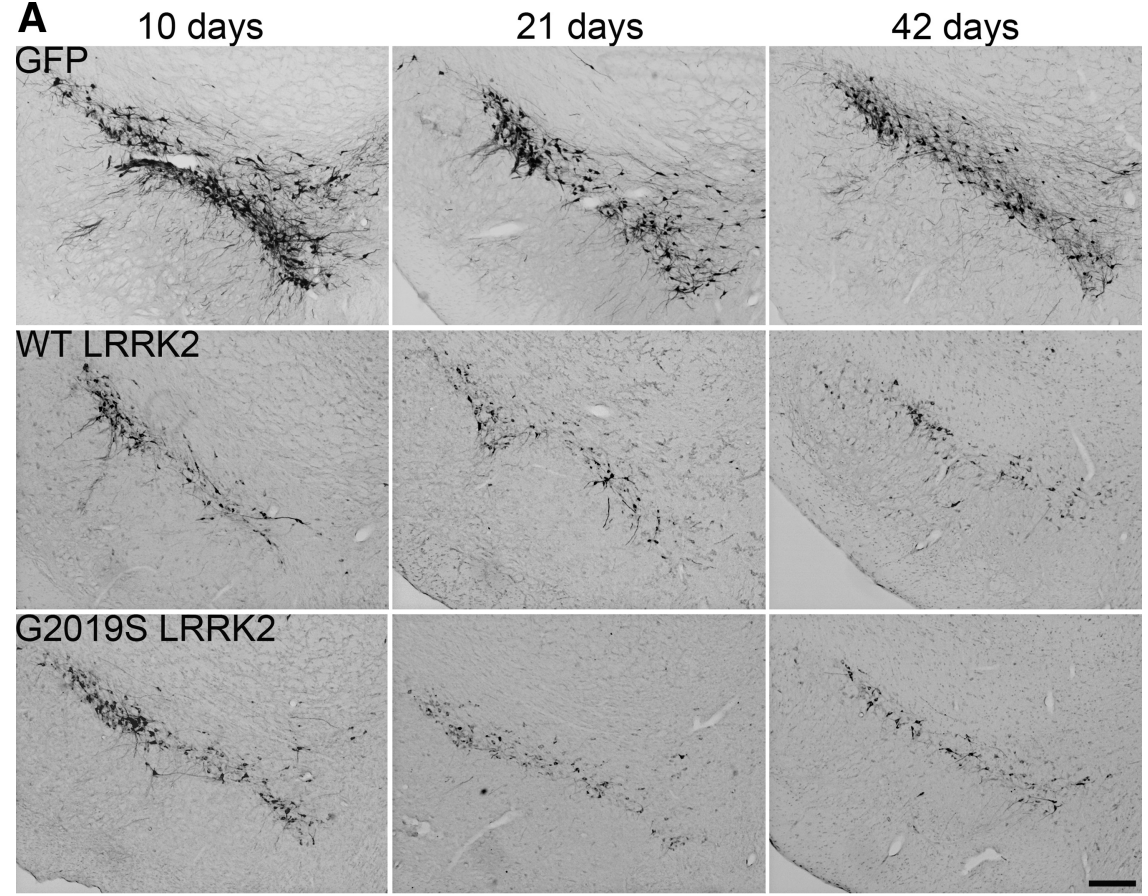

B
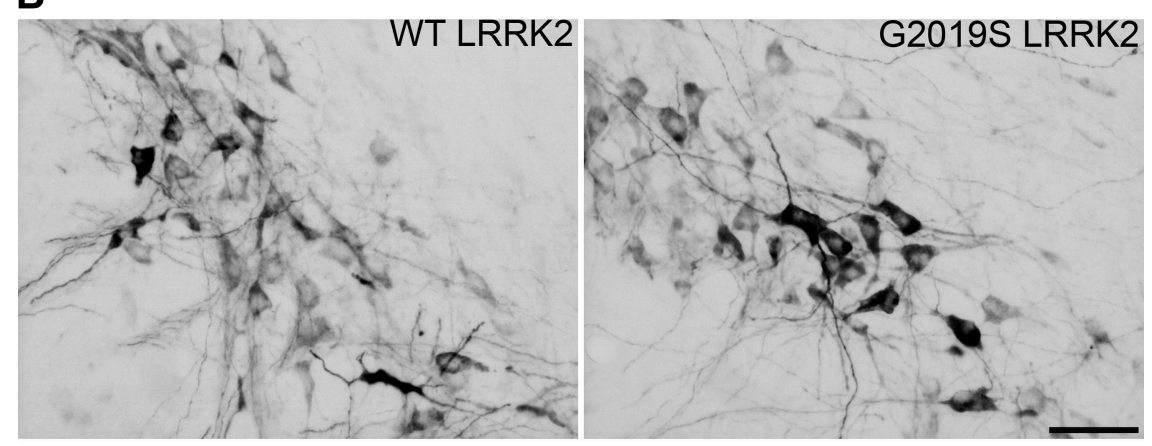

Figure 2. Robust and persistent rAd-driven transgene expression in the SNpc over time. $A$, Representative photomicrographs showing GFP and LRRK2 overexpression, revealed by anti-GFP and anti-FLAG immunostaining, respectively, in retrogradely transduced neurons of the SNpc of animals killed at 10,21, and $42 \mathrm{~d}$ after injection. All animals at each time point displayed robust transgene expression. $\boldsymbol{B}$, High-magnification photomicrographs of anti-FLAG immunostainings in the SNp of rAd-WT-LRRK2-and rAd-G2019S-LRRK2-injected animals at 10 d. Overexpressed LRRK2 localized to the cytoplasm and neuritic processes of transduced nigral neurons. No visible differences in cellular localization were observed between the wild-type and mutant form of overexpressed LRRK2. Scale bars: $\boldsymbol{A}, 200 \mu \mathrm{m} ; \boldsymbol{B}, 50 \mu \mathrm{m}$.

problem during injection). Rats were deeply anesthetized by an overdose of pentobarbital and perfused transcardially first with PBS and then with ice-cold $4 \%$ paraformaldehyde (PFA). Brains were kept for $90 \mathrm{~min}$ in $4 \%$ PFA and then transferred into $25 \%$ sucrose. Twenty-five-micrometerthick coronal sections were harvested on a sliding microtome (SM2400; Leica) at the temperature of $-20^{\circ} \mathrm{C}$. Slices were then processed for immunohistological treatment.

Immunohistochemistry. Primary antibodies used in this study were anti-tyrosine hydroxylase (TH) (rabbit IgG, 1:500; AB152, Millipore Bioscience Research Reagents), anti-GFP (mouse IgG 1:500; A-11120, Invitrogen), anti-FLAG (mouse IgG 1:500; M2 F1804, Sigma), antiVMAT2 (rabbit IgG, 1:2000; AB1767, Millipore Bioscience Research Reagents), anti-ubiquitin (rabbit IgG, 1:1000; Z0458, Dako), anti- $\alpha$ synuclein (mouse IgG, 1:100; Syn-1, BD Biosciences), anti-pS129- $\alpha$ synuclein (mouse IgG, 1:500; pSyn\#64, Wako), anti-Ibal (rabbit IgG, 1:500; 019-19741, Wako), anti-GFAP (mouse IgG, 1:800; G3893, Sigma), and anti-PHF-Tau AT8 (mouse IgG, 1:100, MN1020, AT8 clone, Pierce). For double-fluorescence labeling, we used secondary antibodies conjugated to Alexa Fluor-488 (Invitrogen) and Cy3 (Jackson ImmunoResearch). For bright-field microscopy, we used biotinylated goat anti-rabbit or anti-mouse secondary antibodies (1:200; Vector Laboratories).

Stereological quantification of TH-positive neurons. Unbiased stereological estimation of the number of TH-positive neurons was performed according to the optical fractionator method using the Stereo Investigator Software (MBF Bioscience). Optical dissector grids of $40 \times 40 \mu \mathrm{m}$ were placed in a systematic random manner $100 \times 100 \mu \mathrm{m}$ apart and offset 2 $\mu \mathrm{m}$ from the surface of the section. Gundersen error coefficients of $<0.10$ were accepted. Every eighth section covering the entire extent of the $\mathrm{SNpc}$ was included in the counting procedure. Results were expressed as a percentage loss with respect to the uninjected side. Analyses were performed in a blinded fashion.

Striatal optical density measurement. Striatal dopaminergic innervation at 21 and $42 \mathrm{~d}$ was quantified by measuring the optical density (OD) of TH-immunoreactive terminals in 24 coronal sections (one in six sections) throughout the entire striatum. These sections were scanned using Nikon Super Coolscan 4000 scanner, and the OD of the tissue was analyzed using the NIH ImageJ software.

\section{Statistical analysis}

For comparisons between rAd-GFP, rAd-WTLRRK2, and rAd-G2019S-LRRK2, one-way ANOVAs were performed for each time point (Statistica, Statsoft). Significance was set when $p<0.05$. Data represent mean \pm SEM.

\section{Results}

High-titer recombinant second-generation human serotype 5 adenoviral (rAd) vectors (Zhou and Beaudet, 2000; Young and Neher, 2009) with a sufficiently large packaging capacity for the full-length LRRK2 cDNA ( $\sim 7.6 \mathrm{~kb})$ were generated, encoding GFP, or FLAG-tagged WT or G2019S human LRRK2. Since LRRK2 is predominantly localized to neurons in the mammalian brain (Biskup et al., 2006), the neuronal-specific human synapsin-1 promoter (Kügler et al., 2001) was chosen to drive transgene expression from rAd vectors. Direct injections of rAd in the SNpc results in poor transduction of dopaminergic neurons (Lewis et al., 2010). In contrast, adenoviral particles can be efficiently retrogradely transported to dopaminergic neurons within the SNpc following intrastriatal injections (Kügler et al., 2003). Upon injection of a rAd-GFP vector at six different sites $(2 \mu \mathrm{l}$ per site) in the striatum of adult rats (supplemental Fig. $1 A$, available at www.jneurosci.org as supplemental material), we observed a widespread distribution of rAd-GFP around the injection sites at $10 \mathrm{~d}$ after injection (supplemental Fig. $1 \mathrm{~B}$, available at www. jneurosci.org as supplemental material), resulting in efficient retrograde transport and robust GFP expression in $31 \pm 3.6 \%$ $(n=4)$ of nigral dopaminergic neurons across the entire length of the SNpc (supplemental Fig. 1C, available at www. jneurosci.org as supplemental material), with no visible signs of inflammation in the SNpc (as confirmed by Nissl staining for cellular infiltrations, data not shown). 
rAd-WT-LRRK2 and rAd-G2019S-LRRK2 vectors were shown to drive equivalent levels of expression of LRRK2 in vitro in HEK-293T cells and in vivo in the SNpc and striatum following intrastriatal injections of rats, as described above for rAd-GFP (Fig. 1A). Human LRRK2 is expressed on average at twofold the levels of endogenous LRRK2 as determined from nigral tissue homogenates of injected rats from both WT and G2019S groups (Fig. $1 B$ ). With only $30 \%$ of nigral dopaminergic neurons actually transduced, this finding suggests that high levels of overexpression are achieved in each individual neuron. We further confirmed that human LRRK2 is expressed as an intact full-length protein in vivo by analysis of anti-FLAG immunoprecipitates derived from striatal extracts of rats injected with rAd-WT-LRRK2 and rAd-G2019S-LRRK2 vectors (supplemental Fig. 2, available at www.jneurosci.org as supplemental material). Finally, we demonstrate by native PAGE that endogenous LRRK2 in striatal and nigral extracts assembles into high-molecular-weight complexes ranging from $420 \mathrm{kDa}$ to $>1 \mathrm{MDa}$ (Fig. 1C). Overexpressed WT and G2019S LRRK2 in the striatum and SNpc adopts similar higher-order structures with no obvious difference between LRRK2 variants (Fig. 1C). In native gel conditions, despite loading up to $100 \mu \mathrm{g}$ of total proteins, we could not detect any protein migrating at the size expected for monomeric LRRK2, suggesting that LRRK2 exists predominantly within highmolecular-weight complexes.

Injected animals display robust overexpression of LRRK2 in retrogradely transduced nigral neurons, persisting up to $42 \mathrm{~d}$ after injection despite a progressive decline in expression levels over time (Fig. 2A). As previously reported for first-generation rAd vectors, the observed decline in transgene expression is most likely due to a loss of adenoviral vector genomes in transduced cells (Kügler et al., 2003), since no cell loss is detected in the rAd-GFP and rAd-WT-LRRK2 groups at $42 \mathrm{~d}$ (Fig. 3B). No visible differences in subcellular localization are observed between WT and G2019S LRRK2 (Fig. 2B). Indeed, both forms of the protein localize to the cytoplasm and proximal neuritic processes of nigral dopaminergic neurons, consistent with the expression pattern of endogenous LRRK2 in the SNpc in rodent and human brain (Biskup et al., 2006).

Stereological quantification of the number of dopaminergic neurons in the SNpc was performed at 10, 21, and $42 \mathrm{~d}$ after injection. No cell loss is detected in the rAd-GFP- and rAd-WTLRRK2-injected groups. In contrast, overexpression of G2019S LRRK2 causes a progressive loss of TH-positive dopaminergic neurons in the SNpc, reaching $21.3 \pm 2.2 \%$ at $42 \mathrm{~d}$ (Fig. $3 \mathrm{~A}, \mathrm{~B}$ ). At the same time point, a similar loss of neurons is observed using a second dopaminergic marker, VMAT2 (Fig. 3A,B). Quantification of total Nissl-positive neurons in the SNpc confirms that LRRK2 G2019S causes neuronal cell loss rather than a phenotypic loss of dopaminergic markers (Fig. $3 A, B$ ). In the injected striatum, the presence of an inflammatory reaction around the needle tracts at $10 \mathrm{~d}$ precludes immunostaining at this time point. As of $21 \mathrm{~d}$, however, the inflammation resolves, and an average $10 \%$ decrease in striatal $\mathrm{TH}$-positive fiber density, as determined by striatal OD measurement, is measured for all groups at 21 and $42 \mathrm{~d}$ after injection (supplemental Fig. 3, available at www. jneurosci.org as supplemental material). We do not observe an increased denervation in rAd-G2019S-LRRK2-injected animals relative to the WT-LRRK2 and GFP groups. This may be due to a compensatory sprouting of nigrostriatal projections from the remaining dopaminergic neurons.

Next, we explored the pathological alterations associated with neuronal degeneration due to G2019S LRRK2 overexpression.

\section{A rAd-G2019S-LRRK2}

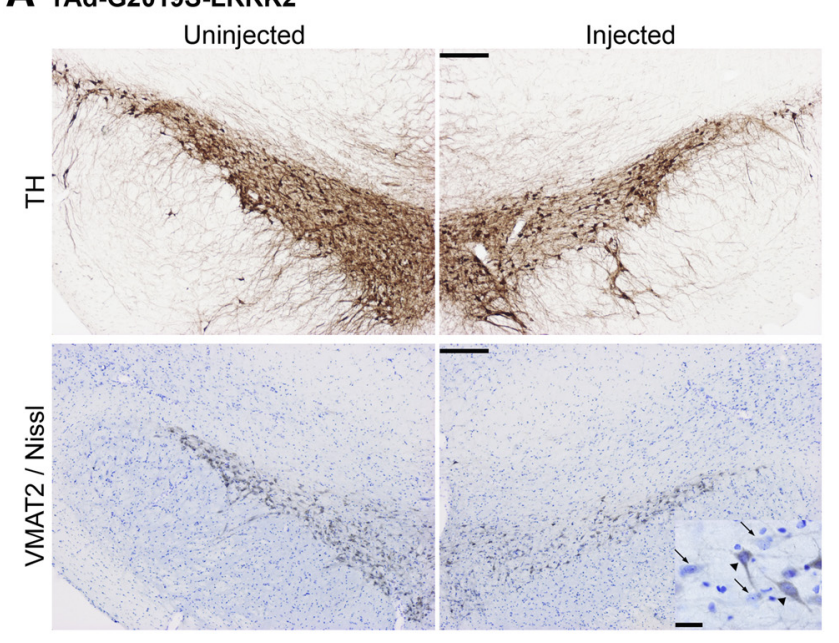

$\mathbf{B}$
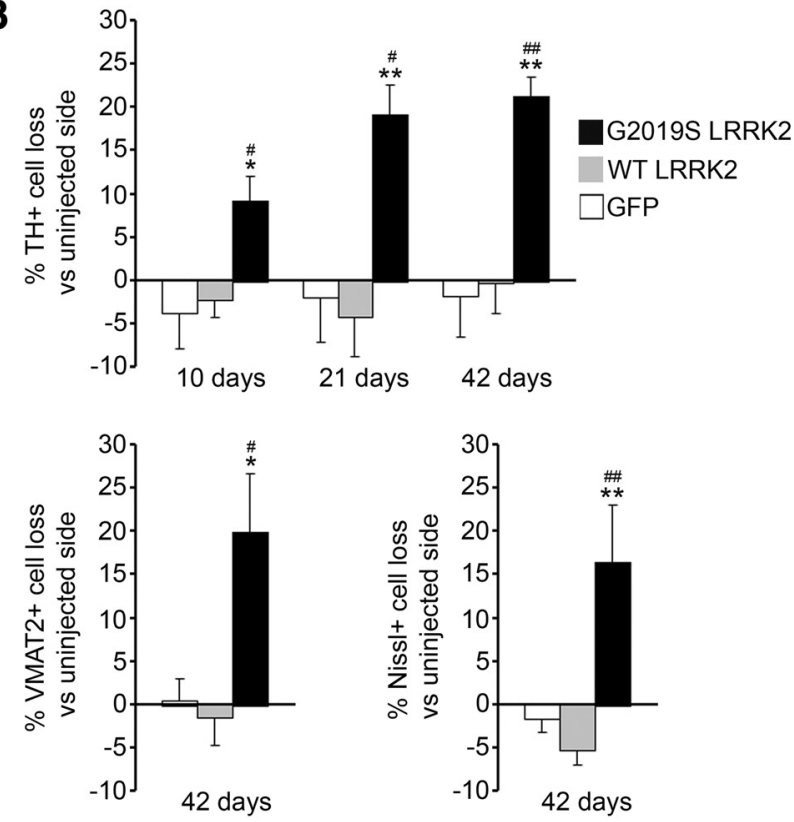

Figure 3. Mutant G2019S LRRK2 causes a loss of nigral dopaminergic neurons. $\boldsymbol{A}$, Representative photomicrographs showing the loss of TH + and VMAT2 + neurons in the SNpc of rAdG2019S-LRRK2-injected animals at $42 \mathrm{~d}$, as revealed by TH and VMAT2 immunohistochemistry. VMAT2-labeled sections were counterstained with NissI to confirm neuronal cell loss. Scale bar, $200 \mu \mathrm{m}$. The higher-magnification inset (scale bar, $20 \mu \mathrm{m}$ ) shows VMAT2+/Nissl+ nigral neurons, indicated by arrowheads, and Niss + nigral neurons, which do not express VMAT2, indicated by arrows. $B$, Stereological quantification of the percentage loss of TH +, VMAT2 +, and NissI + neurons in the SNpc of rAd-GFP-, rAd-WT-LRRK2-, and rAd-G2019S-LRRK2-injected animals ( $n=5 /$ group/time point, except $n=4$ for the G2019S group at 21 d). No significant loss of nigral neurons was detected in the GFP and WT LRRK2 groups. In contrast, G2019S LRRK2 caused a progressive loss of $\mathrm{TH}+$ neurons between 10 and $42 \mathrm{~d}$. Neuronal loss was confirmed by quantification of VMAT2 + and NissI + nigral neurons at 42 d. One-way ANOVA statistics for the loss of TH + nigral neurons at $10 \mathrm{~d}, F_{(2,12)}=4.96 ; 21 \mathrm{~d}, F_{(2,11)}=6.82$; and $42 \mathrm{~d}, F_{(2,12)}=8.66$; for the loss of VMAT2 + nigral neurons at $42 \mathrm{~d}, F_{(2,12)}=6.44$; for the loss of Nissl + nigral neurons at $42 \mathrm{~d}, F_{(2,12)}=7.88$. Newman-Keuls post hoc analyses showed significant differences between the G2019S-LRRK2 and GFP groups $\left({ }^{*} p<0.05,{ }^{* *} p<0.01\right)$, and the G2019SLRRK2 and WT-LRRK2 groups ( $\left.{ }^{\#} p<0.05,{ }^{\#} p<0.01\right)$. There were no significant differences between the GFP and WT-LRRK2 groups. Bars represent the mean \pm SEM.

We do not detect abnormal accumulation or aggregation of $\alpha$-synuclein and ubiquitin in dopaminergic neurons of the injected SNpc in either group up to $42 \mathrm{~d}$ (supplemental Fig. 4, available at www.jneurosci.org as supplemental material). Nor does LRRK2 overexpression lead to any increase in endogenous 


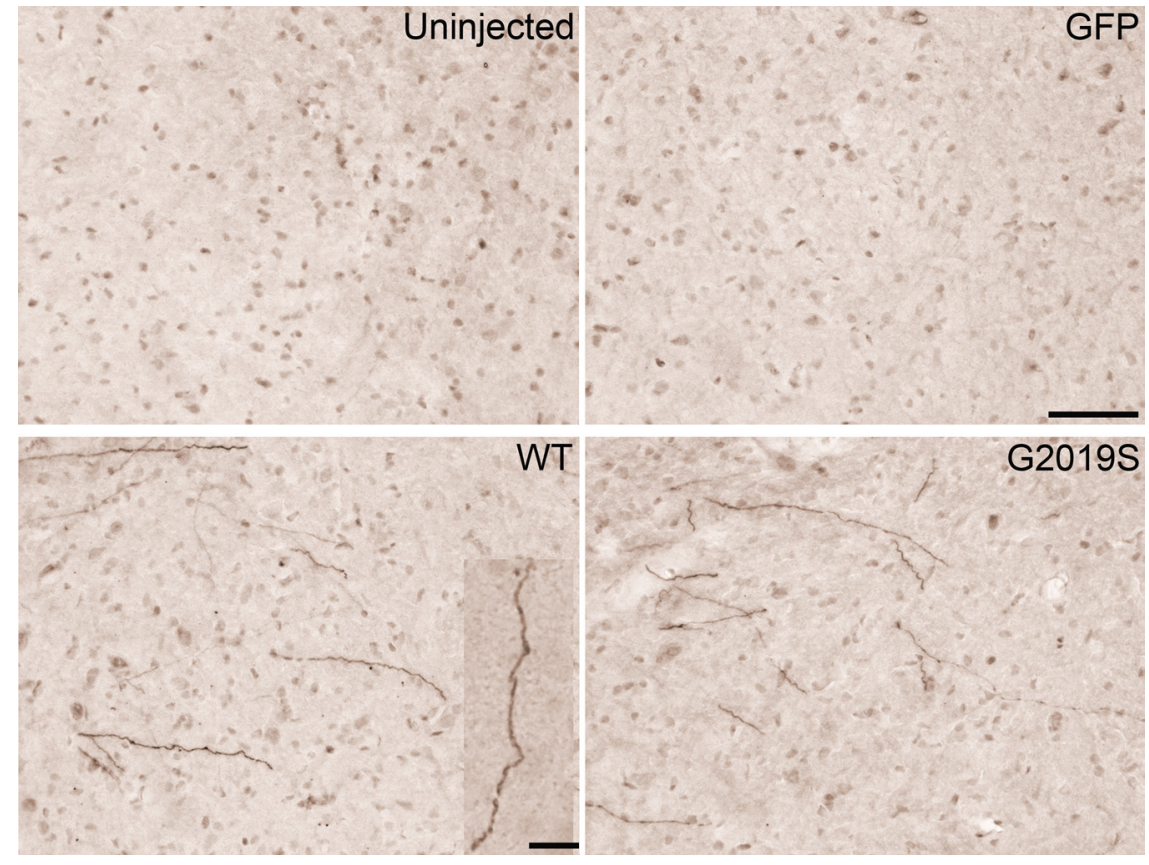

Figure 4. Abnormal transient hyperphosphorylation of tau in dystrophic nigral neuritic processes upon LRRK2 overexpression at $10 \mathrm{~d}$. Immunohistochemistry with AT8 antibody revealed abnormal hyperphosphorylation of tau in neuritic processes of the SNpc of rAd-WT-LRRK2- and rAd-G2019S-LRRK2-injected animals at $10 \mathrm{~d}$. AT8-positive neurites often appeared fragmented with a beaded morphology (see inset in lower left panel). We counted an average of $24 \pm 8(n=3)$ and $25 \pm 5(n=3)$ AT8-positive neurites per $25-\mu \mathrm{m}$-thick section per animal in the $\mathrm{G} 2019 \mathrm{~S}$ and WT groups, respectively. Scale bar, $50 \mu \mathrm{m}$; inset, $10 \mu \mathrm{m}$. mutations in LRRK2 (Li et al., 2009, 2010; Lin et al., 2009; Tong et al., 2009; Melrose et al., 2010). The difference in phenotype with the present model may be explained by higher levels of LRRK2 expression achieved with viral vectors. Compensatory mechanisms may also have been activated in transgenic mice, linked to the onset of expression during development. Nigral neurodegeneration induced by G2019S LRRK2 was also recently demonstrated in mice, using HSV amplicon as a gene transfer tool (Lee et al., 2010), further supporting the use of viral vectors to model LRRK2 pathology in rodents.

In summary, we have generated a new rat model of $\mathrm{PD}$, based on the most common PD-associated mutation in LRRK2, which recapitulates one of the cardinal pathological features of the disease. We believe this viral gene-transfer model will be a highly valuable tool for the in vivo evaluation of neuroprotective therapies for both familial and sporadic PD.

\section{References}

Berg D, Schweitzer K, Leitner P, Zimprich A, Lichtner P, Belcredi P, Brussel T, Schulte C, Maass S, Nagele T (2005) Type and frequency of mutations in the LRRK2 gene in $\alpha$-synuclein phosphorylation at residue serine 129 (supplemental Fig. 4, available at www.jneurosci.org as supplemental material). However, immunostaining for the pathological phospho-tau epitope (pSer202/pThr205) with the AT8 antibody reveals abnormal hyperphosphorylation of tau in dystrophic nigral neuritic processes upon WT and G2019S LRRK2 overexpression, but not GFP, at $10 \mathrm{~d}$ after injection (Fig. 4). AT8-positive immunostaining is no longer visible at $21 \mathrm{~d}$ after injection (supplemental Fig. 5, available at www.jneurosci.org as supplemental material), suggesting a transient appearance correlating with maximal expression of WT or G2019S human LRRK2, but not correlating with neuronal cell death, present only with G2019S LRRK2 transduction. We do not detect any AT8-immunopositive processes in the striatum at $21 \mathrm{~d}$ (supplemental Fig. 5, available at www.jneurosci. org as supplemental material). When compared to the GFPexpressing animals, either WT or G2019S LRRK2 expression does not lead to increased recruitment and activation of microglia and astrocytes as measured by Ibal and GFAP immunostaining in the striatum and SNpc (data not shown).

\section{Discussion}

Adenoviral vectors provide an efficient answer to the size constraints of the LRRK2 coding sequence $(\sim 7.6 \mathrm{~kb})$ and represent highly flexible tools for the study of mutant LRRK2 pathogenesis in the SNpc of adult rats. By introducing an abrupt change in LRRK2 expression in the adult brain, we induce a robust and rapidly progressing nigral neurodegeneration. This technology also enables direct comparisons between the wild-type and G2019S variants of LRRK2, allowing us to unambiguously establish that LRRK2 causes progressive nigral neurodegeneration only in the presence of the G2019S mutation.

Existing LRRK2 transgenic mice, in contrast, do not display nigral cell loss and likely represent models of early functional impairments and pathogenic events arising from PD-associated familial and sporadic Parkinson's disease ${ }^{\star}$. Brain 128:3000-3011.

Biskup S, Moore DJ, Celsi F, Higashi S, West AB, Andrabi SA, Kurkinen K, Yu SW, Savitt JM, Waldvogel HJ, Faull RL, Emson PC, Torp R, Ottersen OP, Dawson TM, Dawson VL (2006) Localization of LRRK2 to membranous and vesicular structures in mammalian brain. Ann Neurol 60:557-569.

Di Fonzo A, Rohé CF, Ferreira J, Chien HF, Vacca L, Stocchi F, Guedes L, Fabrizio E, Manfredi M, Vanacore N, Goldwurm S, Breedveld G, Sampaio C, Meco G, Barbosa E, Oostra BA, Bonifati V, the Italian Parkinson Genetics Network (2005) A frequent LRRK2 gene mutation associated with autosomal dominant Parkinson's disease. Lancet 365:412-415.

Giasson BI, Covy JP, Bonini NM, Hurtig HI, Farrer MJ, Trojanowski JQ, Van Deerlin VM (2006) Biochemical and pathological characterization of Lrrk2. Ann Neurol 59:315-322.

Gilks WP, Abou-Sleiman PM, Gandhi S, Jain S, Singleton A, Lees AJ, Shaw K, Bhatia KP, Bonifati V, Quinn NP, Lynch J, Healy DG, Holton JL, Revesz T, Wood NW (2005) A common LRRK2 mutation in idiopathic Parkinson's disease. Lancet 365:415-416.

Healy DG, Falchi M, O'Sullivan SS, Bonifati V, Durr A, Bressman S, Brice A, Aasly J, Zabetian CP, Goldwurm S, Ferreira JJ, Tolosa E, Kay DM, Klein C, Williams DR, Marras C, Lang AE, Wszolek ZK, Berciano J, Schapira AH, et al. (2008) Phenotype, genotype, and worldwide genetic penetrance of LRRK2-associated Parkinson's disease: a case-control study. Lancet Neurol 7:583-590.

Kügler S, Meyn L, Holzmüller H, Gerhardt E, Isenmann S, Schulz JB, Bähr M (2001) Neuron-specific expression of therapeutic proteins: evaluation of different cellular promoters in recombinant adenoviral vectors. Mol Cell Neurosci 17:78-96.

Kügler S, Kilic E, Bähr M (2003) Human synapsin 1 gene promoter confers highly neuron-specific long-term transgene expression from an adenoviral vector in the adult rat brain depending on the transduced area. Gene Ther 10:337-347.

Lee BD, Shin JH, VanKampen J, Petrucelli L, West AB, Ko HS, Lee YI, Maguire-Zeiss KA, Bowers WJ, Federoff HJ, Dawson VL, Dawson TM (2010) Inhibitors of leucine-rich repeat kinase-2 protect against models of Parkinson's disease. Nat Med 16:998-1000.

Lesage S, Dürr A, Tazir M, Lohmann E, Leutenegger AL, Janin S, Pollak P, Brice A (2006) LRRK2 G2019S as a cause of Parkinson's disease in North African Arabs. N Engl J Med 354:422-423. 
Lewis TB, Glasgow JN, Glandon AM, Curiel DT, Standaert DG (2010) Transduction of brain dopamine neurons by adenoviral vectors is modulated by CAR expression: rationale for tropism modified vectors in PD gene therapy. PloS One 5:e12672.

Li X, Patel JC, Wang J, Avshalumov MV, Nicholson C, Buxbaum JD, Elder GA, Rice ME, Yue Z (2010) Enhanced striatal dopamine transmission and motor performance with LRRK2 overexpression in mice is eliminated by familial Parkinson's disease mutation G2019S. J Neurosci 30:1788-1797.

Li Y, Liu W, Oo TF, Wang L, Tang Y, Jackson-Lewis V, Zhou C, Geghman K, Bogdanov M, Przedborski S, Beal MF, Burke RE, Li C (2009) Mutant LRRK2(R1441G) BAC transgenic mice recapitulate cardinal features of Parkinson's disease. Nat Neurosci 12:826-828.

Lin X, Parisiadou L, Gu XL, Wang L, Shim H, Sun L, Xie C, Long CX, Yang WJ, Ding J, Chen ZZ, Gallant PE, Tao-Cheng JH, Rudow G, Troncoso JC, Liu Z, Li Z, Cai H (2009) Leucine-rich repeat kinase 2 regulates the progression of neuropathology induced by Parkinson's-disease-related mutant alpha-synuclein. Neuron 64:807-827.

Melrose HL, Dächsel JC, Behrouz B, Lincoln SJ, Yue M, Hinkle KM, Kent CB, Korvatska E, Taylor JP, Witten L, Liang YQ, Beevers JE, Boules M, Dugger BN, Serna VA, Gaukhman A, Yu X, Castanedes-Casey M, Braithwaite AT, Ogholikhan S, et al. (2010) Impaired dopaminergic neurotransmission and microtubule-associated protein tau alterations in human LRRK2 transgenic mice. Neurobiol Dis 40:503-517.

Ng P, Graham FL (2002) Construction of first-generation adenoviral vectors. Methods Mol Med 69:389-414.

Ozelius LJ, Senthil G, Saunders-Pullman R, Ohmann E, Deligtisch A, Tagliati M, Hunt AL, Klein C, Henick B, Hailpern SM, Lipton RB, Soto-Valencia J, Risch N, Bressman SB (2006) LRRK2 G2019S as a cause of Parkinson's disease in Ashkenazi Jews. N Engl J Med 354:424-425.

Ross OA, Toft M, Whittle AJ, Johnson JL, Papapetropoulos S, Mash DC, Litvan I, Gordon MF, Wszolek ZK, Farrer MJ, Dickson DW (2006) Lrrk2 and Lewy body disease. Ann Neurol 59:388-393.

Tong Y, Pisani A, Martella G, Karouani M, Yamaguchi H, Pothos EN, Shen J (2009) R1441C mutation in LRRK2 impairs dopaminergic neurotransmission in mice. Proc Natl Acad Sci U S A 106:14622-14627.

Young SM Jr, Neher E (2009) Synaptotagmin has an essential function in synaptic vesicle positioning for synchronous release in addition to its role as a calcium sensor. Neuron 63:482-496.

Zhou H, Beaudet AL (2000) A new vector system with inducible E2a cell line for production of higher titer and safer adenoviral vectors. Virology 275: 348-357. 